\title{
Deep segmentation of the liver and the hepatic tumors from abdomen tomography images
}

\author{
Nermeen Elmenabawy ${ }^{1}$, Mervat El-Seddek ${ }^{2}$, Hossam El-Din Moustafa ${ }^{1}$, Ahmed Elnakib $^{1}$ \\ ${ }^{1}$ Department of Electronics and Communications Engineering, Faculty of Engineering, Mansoura University, Mansoura, Egypt \\ ${ }^{2}$ Department of Communications and Electronics Engineering, Misr Higher Institute of Engineering and Technology, Mansoura, Egypt
}

\begin{tabular}{l} 
Article Info \\
\hline Article history: \\
Received Dec 1, 2020 \\
Revised Aug 3, 2021 \\
Accepted Aug 14, 2021 \\
\hline Keywords: \\
Computed tomography \\
Deep learning \\
Liver \\
Segmentation \\
Tumors
\end{tabular}

\begin{abstract}
A pipelined framework is proposed for accurate, automated, simultaneous segmentation of the liver as well as the hepatic tumors from computed tomography (CT) images. The introduced framework composed of three pipelined levels. First, two different transfers deep convolutional neural networks (CNN) are applied to get high-level compact features of CT images. Second, a pixel-wise classifier is used to obtain two outputclassified maps for each CNN model. Finally, a fusion neural network (FNN) is used to integrate the two maps. Experimentations performed on the MICCAI'2017 database of the liver tumor segmentation (LITS) challenge, result in a dice similarity coefficient (DSC) of $93.5 \%$ for the segmentation of the liver and of $74.40 \%$ for the segmentation of the lesion, using a 5-fold cross-validation scheme. Comparative results with the state-of-the-art techniques on the same data show the competing performance of the proposed framework for simultaneous liver and tumor segmentation.
\end{abstract}

This is an open access article under the CC BY-SA license.

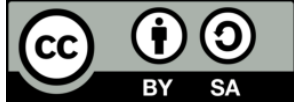

\section{Corresponding Author:}

Ahmed Elnakib

Department of Electronics and Communications Engineering, Faculty of Engineering

Mansoura University

Elgomhouria St., Mansoura 35516, Egypt

Email: nakib@mans.edu.eg

\section{INTRODUCTION}

According to the World Health Organization (WHO), liver cancer is the main cause of cancer deaths among all types of cancers. Worldwide, around 800,000 cases of liver cancer are diagnosed each year, accounting for around 700,000 deaths [1]. In 2019, the American Cancer Society (ACS) estimated around 42,030 new cases for primary liver cancer and intrahepatic bile duct cancer in the United States, with around 31,780 deaths [1]. These metrics reflect the epidemic inflation of liver cancer.

Computed tomography (CT) imaging is usually used for liver segmentation and/or liver cancer detection. However, manual segmentation of the liver and/or the liver tumors from CT images consumes a lot of time and suffers from observer variability. Therefore, the design of efficient computer aided diagnostic (CAD) systems, to assist the radiologists for liver segmentation and/or liver cancer segmentation, is a widely investigated open research problem. Throughout literature, different methodologies have been utilized for liver segmentation and/or for liver cancer segmentation. These methods can be categorized as traditional methods or deep learning methods.

Traditional approaches usually extract features, e.g., intensity, texture, shape, from liver CT images and use a classifier based on these features to perform the segmentation process. On the other hand, deep learning methods usually use convolutional neural networks (CNN) that consist of a number of convolutional layers for extracting low-level and high-level features for the liver CT images and fully connected layers to encode a compact feature set for the segmentation process. 
For the task of liver segmentation, a preliminary step in many CAD systems for liver cancer [2] and liver fibrosis [3], different traditional and deep learning methods have been applied. For example, Barstugan et al. [4] used a super-pixel linear iterative clustering approach and AdaBoost algorithm to segment the liver, achieving a DSC of $92.13 \%$ on 16 abdomen CT test images. Muthuswamy and Kanmani [5] extracted the liver from CT images based on intensity thresholding, fuzzy c-means clustering, and connected component analysis. For example, Chang et al. [6] segmented the tumors using a region growing algorithm. A binary logistic regression analysis based on extracted texture, shape, and kinetic curve features were further performed to classify the segmented tumors (Benign or Malignant). In Chlebus et al. [7], a modified U-ne [8] architecture was used, consists of four resolution levels, for liver tumors segmentation. Yuan [9] used a hierarchical deep fully convolutional-deconvolutional neural networks (CDNN) for tumor segmentation. An initial liver segmentation was provided using a simple CDNN model. The segmented liver region was refined using another CDNN to find the final liver segmentation enhanced by histogram equalization. Then a third CDNN is applied for tumor segmentation. Bi et al. [10] used a deep residual networks (ResNet) for liver and lesions segmentation. Gruber et al. [11] applied, sequentially, two U-net [8] networks for liver and lesions segmentation. Wang et al. [12] a 3D atlas-based model for liver segmentation. Shi et al. [13] utilized a deformable shape liver segmentation method. Song et al. [14] implemented a modified U-Net model for liver segmentation. Although the methods presented in the literature achieved good results, the accuracy is still a need to be improved. The present study presents a deep learning system for simultaneous liver and tumor segmentation using CNN modeling. The main contributions of this work are as follows:

- Investigating different deep learning architectures for liver and tumor segmentation (i.e., Densenet and FCN-AlexNet)

- Applying a 3D narrow-band of the input images to enhance the deep training

- Using a smart fusion of two CNN architectures to improve the segmentation quality

- $\quad$ Performance evaluation on the MICCAI'2017 challenge liver tumor segmentation (LITS) database.

The structure of this paper is as following. Section 2 presents the suggested system for simultaneous liver and tumor segmentation. Section 3 summarizes the proposed system results as well as the comparative results to the current state-of-the-art techniques. Finally, section 4 concludes the paper.

\section{METHODS}

The proposed framework processes a raw image through three stages as shown in Figure 1. First, features are extracted from raw images, without preprocessing steps, by investigating two different CNN models. Second, a pixel-wise classification layer is applied. Finally, a smart fusion of the outputs of the two $\mathrm{CNN}$ models is performed using a neural network (NN) to provide the final simultaneous liver and tumor segmentation map, containing three output labels: background (BG), liver, and lesion.

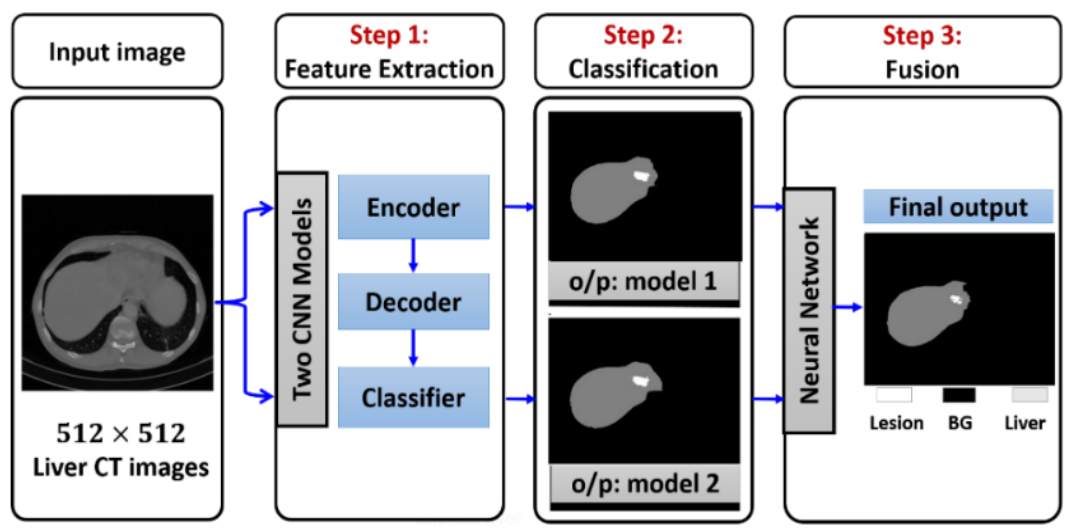

Figure 1. Proposed framework for the liver and lesions segmentation with three stages: feature extraction using deep learning, classification based on pixel-wise technique, and smart fusion

\subsection{Feature extraction}

Herein, two pre-trained CNNs are used to get the features of the liver and its lesions; Densenet [15] and the fully connected network (FCN) using Alexnet (FCN-Alexnet [16]). The Densenet model consists of a down-sampling path and up-sampling path. The down-sampling path extracted the semantic features then the 
up-sampling path is trained to recover the image resolution of the input at the output of the model. Figure 2 shows the architecture of the Densenet model.

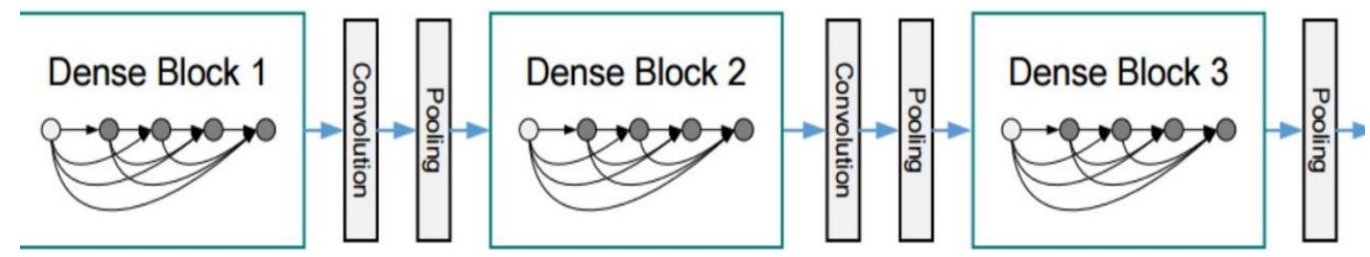

Figure 2. Schematic diagrams for the structures of Densnet model [15]

FCN-Alexnet consists of an encoder, a decoder, and a pixel-wise classifier as shown in Figure 3. The job of the encoder is to extract high-level compact deep learning features from the abdomen liver CT images. For the FCN-Alexnet model, the stage of the encoder consists of five Alexnet's layers as shown in Table 1, with no fully connected layers as shown in Figure 3. The job of the decoder is to perform deconvolutional steps to get extracted features with the same dimensions as the input image. To perform the segmentation process, a classification layer based on pixel-wise technique is further used, to label each pixel in the CT input image into one of three labels: lesion, liver, or background.

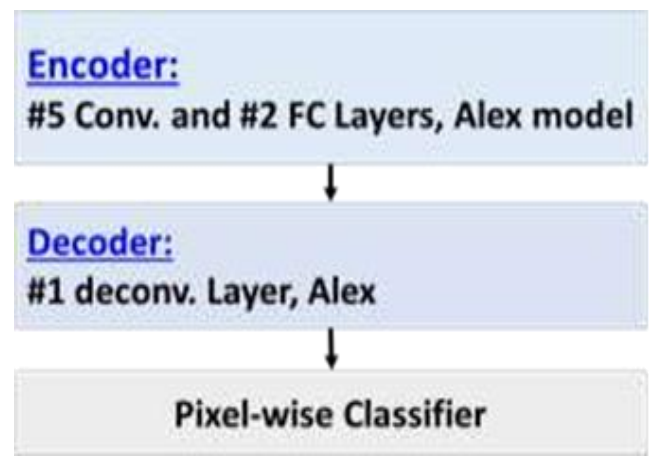

Figure 3. Schematic diagrams for the structures of FCN-Alexnet [16]

The pre-trained Densenet and Alexnet are trained on the ImageNet large-scale visual recognition challenge 2012 (ILSVRC2012) dataset that is composed of 10 million training images of size $224 \times 224$ from more than 1000 subjects. The Densenet network composed of contiguous dense blocks. There are a transition layers (convolutional layers and average pooling) between the contiguous dense blocks with more than 20 million parameters. The size of the feature map and the dense block is similar to be concatenated easily. There are a global average pooling and a SoftMax classifier at the end of the last dense block. Alexnet model is composed of five convolutional layers and three FC layers with more than 62.4 million parameters. More detailed of each model can be found in [15], [16], respectively. We applied the two models (Densenet and FCN-Alexnet) in the proposed system, since their decoders produce outputs that are of the same dimensions as the input image, which suits the task of segmentation. In addition, they have shown outstanding performance for several related medical applications, such as lung segmentation [17], [18], pulmonary cancerous detection [19], face recognition [20], brain cancer [21] and diabetic retinopathy [22].

\subsection{Classification}

A pixel-wise classifier is applied after each model's decoder to label the segmented output image. The pixel-wise classifier is composed of two layers: a SoftMax layer and a weighted layer to perform pixelwise classification. The SoftMax layer is composed of three SoftMax nodes per each image pixel, providing the probabilities of the three labels: lesion, liver, or background, as in (1).

$$
\sigma\left(x_{i}\right)=\frac{e^{x_{i}}}{\sum_{i} e^{x_{i}}}
$$


where $x_{i}$ denotes the input at the softmax node $i$ and $\sigma($.$) denotes the output probability of the SoftMax node.$ The weights of the pixel classification layer are trained using the LITS database. Based on the largest SoftMax probability, the pixel-wise classification layer provides the final output label for each pixel to be either lesion, liver, or background.

\subsection{Fusion neural network (FNN)}

To investigate the potential of fusing the extracted deep learning features from the two utilized deep learning models (Densenet and FCN-Alexnet), an FNN is designed to integrate the strength of each model. The proposed FNN consists of an input layer, one fully connected hidden layer, and an output layer as shown in Figure 4. The input layer of the FNN consists of the two input labeled images (from the outputs of the Densenet and the FCN-Alexnet models). The hidden layer is composed of a number of $H 1$ nodes, $H 1=100$, selected during experimentations, all with tanh activation functions. The output layer is composed of the finally fused output labeled image with the same dimensions as the input images. Figure 4 shows a typical example of fusion, where the proposed FNN was able to enhance the performance of the given example.

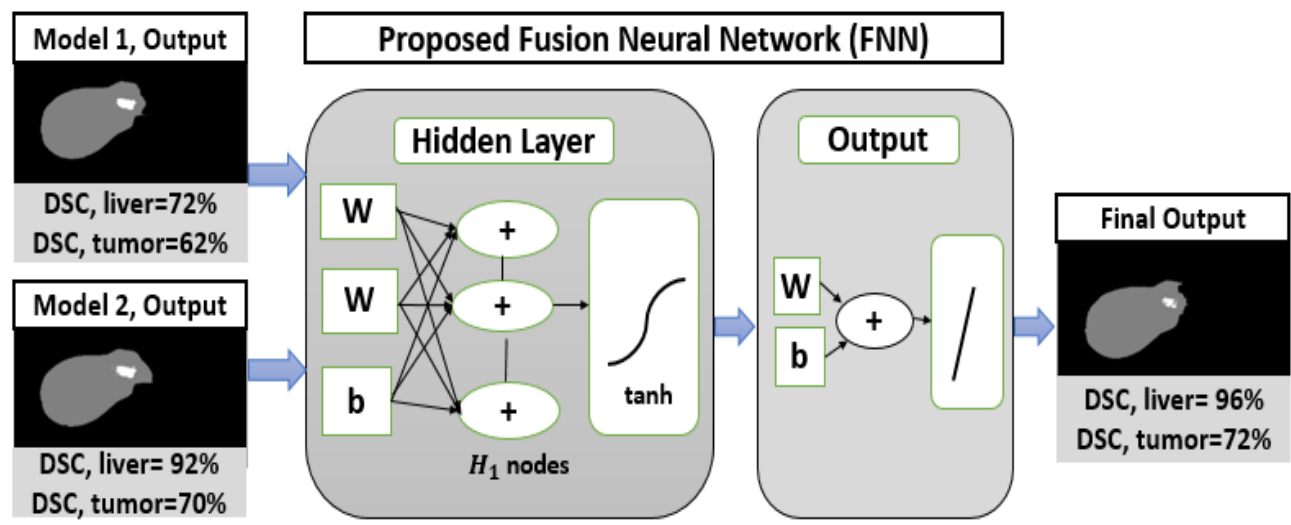

Figure 4. Architecture of the proposed FNN

\subsection{Performance metrics}

In order to accurately evaluate the performance of the proposed system for the liver and tumor segmentation, two parameters are used to assess the quality of segmentation: one area-based metric; the dice similarity coefficient (DSC), and a distance-based metric; the average symmetric surface distance (ASSD). The DSC [23] represents the area overlap between the segmented image (S) and the ground truth (GT) image:

$$
\operatorname{DSC}(S, G T)=|S \cap G T| / 0.5(|S|+|G T|) \times 100 \%
$$

where the $|$.$| operator denotes the object area.$

On the other hand, the ASSD [24] measures the distance between the segmented object surface and its corresponding GT segmentation surface, known as the average of the Euclidian distances, $d$, from (i) all points, $x$, on the surface of the segmented object ( $S s)$ to the surface of the GT (GTs) and (ii) all points on the GTs to $S s$ :

$$
\operatorname{ASSD}(S, G T)=1 /(|S s|+|G T s|) \times\left(\sum_{x \in S S}(\mathrm{x}, \mathrm{GTs})+\sum_{\mathrm{x} \in \mathrm{GTs}}(\mathrm{x}, \mathrm{Ss})\right) \times 100 \%
$$

\section{EXPERIMENTAL RESULTS AND DISCUSSION}

In this section, the LITS challenging database, the experimental setup, and the comparative results to other methods are detailed.

\subsection{LITS database}

The LITS challenging database [25], [26] consists of 130 contrast-enhanced abdominal CT training scans collected from seven different clinical institutions. The training CT scans were given with manual segmentations of the liver and liver lesions done by trained radiologists. All volumes contained a different 
number of axial slices (42 to 1026 cross-section per volume), with an overall number of 16,917 images. The size of each CT image is $512 \times 512$ pixels. Data description is detailed in [25] and [26].

\subsection{Experimental setting}

Model 1 (Densenet) and Model 2 (FCN-Alexnet) are trained using the database of LITS competition as follows: initially, all the encoder's weights are initialized by transferring the Densenet network in [15] and Alexnet in [16] pertained weights, respectively. In the training phase, all encoder layers and decoder layers are fine-tuned using the LITS data. The training epochs are repeated until the crossentropy loss is very small or the number of epochs exceeds 30 . Inputs are shuffled in each epoch using a mini-patch size of 500. Learning rates are set to $10^{-3}$ for model 1 and for model 2 to afford higher parameter tuning. FNN training applied the same training setting. All training phases are implemented using MATLAB@ 2018a. Over-fitting is avoided by reducing the network's capacity by removing layers (fully connected layer in the pre-trained network Alexnet in model 2) and reducing the number of elements in the hidden layers in the fusion network.

A Five-fold cross-validation is used to evaluate the proposed system. Two modes are used for the input data: "duplicate" and "3D narrow-band" as shown in Figure 5. In the "Duplicate" mode, the input data is composed of three duplicated grey level images at each of the three standard channels of the utilized deep learning model. In the "3D narrow-band" mode, input data is composed of three consequent anatomical grey level images to the proposed system (i.e., the target image to the centralized CNN model's input channel and the previous and next cross-sections to each side channel).

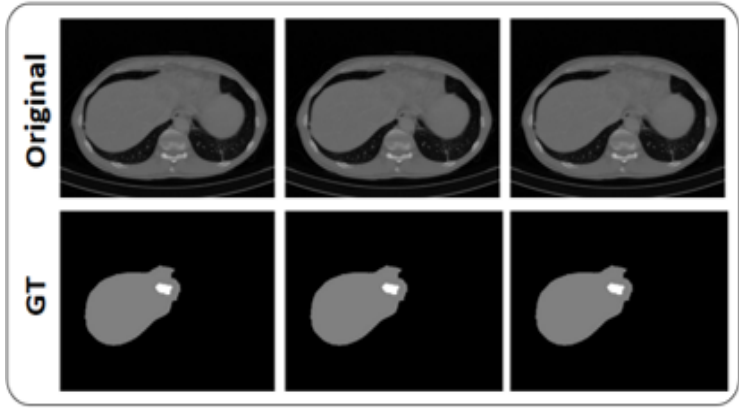

(a)

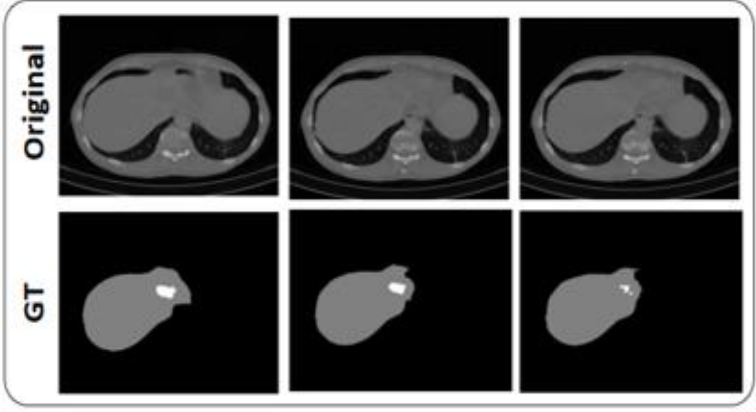

(b)

Figure 5. The data are input to the proposed system using two modes: (a) "Duplicate" and (b) "3D Narrowband". Original image (top row) and GT image (bottom row)

A five-fold cross-validation is applied to evaluate the proposed system with two different settings: "global" and "per case". The "global" setting applies the 5-fold cross validation on the whole 16,917 images of all the 130 scans (i.e., 383 test images (20\% of images) and 13,534 training images (80\% of the images)). On the other side, the "per case" setting divide the data based on case (subject or scan) and applies the 5-fold cross-validation on the total number of 130 separate scans (i.e., 26 test subjects' images (20\% of the scans) and 104 training subjects ( $80 \%$ of the scans)). Cross-entropy is used as the objective function to train the network using ADAM optimizer [27]. The median frequency balancing is used, where the weight assigned to a class in the loss function.

\subsection{Experimental results}

In order to assess quantitatively the system performance, Table 1 provides detailed liver and tumor segmentation results for each utilized CNN model (Densenet and FCN- Alexnet) as well as the proposed fused system. Consistent with the visual results in Figure 6, the performance of FCN-Alexnet model is better than the Densenet network. This is due to the efficient simpler structure of the FCN-Alexnet (its encoder contains only five convolutional layers plus 2 fully connected layers, which is easy to be trained efficiently) compared to the Densenet (contains 201 layers [15], making its training rather complex and causes overfitting). In addition, Tables 1, 2, and Figure 6 show that the proposed FNN fusion further improves the performance. As expected, the "3D Narrow-band" mode achieves better results than the "Duplicate" mode, since it takes into account an extended 3D narrow-band anatomical information of the object. However, Table 1 shows that while the "3D Narrow-band" mode achieves better results for tumor segmentation for all the three compared systems (Densenet, FCN-Alexnet, and the proposed system), it fails to enhance the liver 
segmentation results. This is due to the almost no significant change between the liver anatomies for the consequent images, while tumor anatomy shows significant changes due to its relatively small size compared with the liver.

Table 1. Liver and tumor segmentation results for each utilized deep learning model (Densenet, FCN-

Alexnet, and FNN). For each model, results are compared for two modes

\begin{tabular}{|c|c|c|c|c|c|c|c|c|c|}
\hline \multirow[t]{3}{*}{ Model } & \multirow{3}{*}{$\begin{array}{l}\text { Object } \\
\text { Mode } \\
\text { Metric }\end{array}$} & \multicolumn{4}{|c|}{ Liver } & \multicolumn{4}{|c|}{ Tumor } \\
\hline & & \multicolumn{2}{|c|}{ “Global” } & \multicolumn{2}{|c|}{ "Per case" } & \multicolumn{2}{|c|}{ "Global" } & \multicolumn{2}{|c|}{ "Per case" } \\
\hline & & DSC & ASSD & DSC & ASSD & DSC & ASSD & DSC & ASSD \\
\hline Model 1 & Duplicate & $82.8 \%$ & 3.89 & $76.5 \%$ & 4.95 & $69.7 \%$ & 3.87 & $62.6 \%$ & 4.12 \\
\hline Densenet & Narrow-band 3D & $82.8 \%$ & 3.89 & $76.5 \%$ & 4.95 & $73.0 \%$ & 2.76 & $64.8 \%$ & 3.07 \\
\hline Model 2 & Duplicate & $96.9 \%$ & 0.89 & $91.4 \%$ & 1.32 & $76.3 \%$ & 3.21 & $66.1 \%$ & 3.87 \\
\hline FCN Alexnet & Narrow-band 3D & $96.9 \%$ & 0.85 & $91.4 \%$ & 1.35 & $78.2 \%$ & 2.43 & $68.9 \%$ & 3.18 \\
\hline Proposed: & Duplicate & $97.2 \%$ & 0.74 & $93.5 \%$ & 0.99 & $78.8 \%$ & 2.36 & $70.0 \%$ & 3.11 \\
\hline FNN fusion & Narrow-band 3D & $97.2 \%$ & 0.72 & $93.5 \%$ & 0.77 & $79.9 \%$ & 0.92 & $\mathbf{7 4 . 4 \%}$ & 0.99 \\
\hline
\end{tabular}

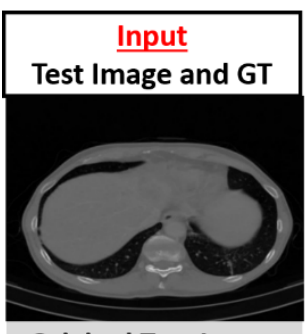

Original Test Image

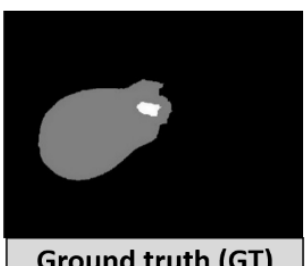

Ground truth (GT)

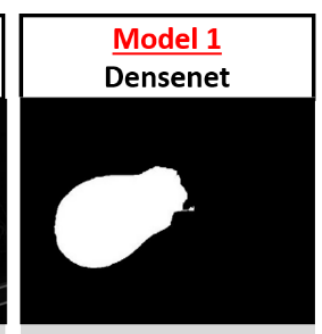

Dice, liver $=\mathbf{8 2} \%$

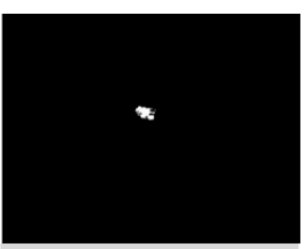

Dice, tumor $=69 \%$

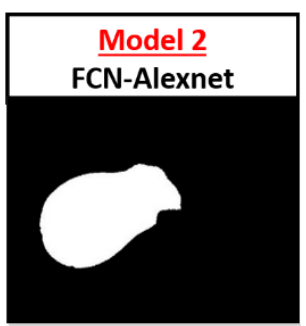

Dice, liver $=91 \%$

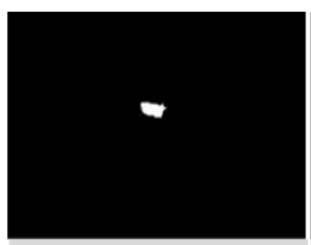

Dice, tumor $=\mathbf{7 2} \%$

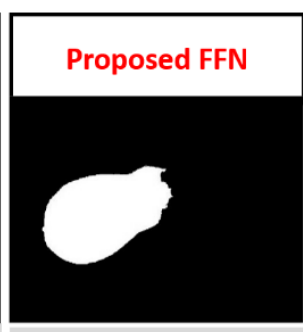

Dice, liver $=93 \%$

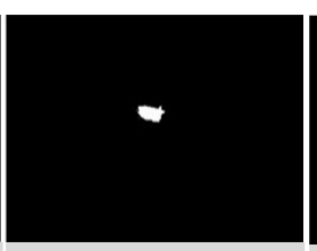

Dice, tumor $=74 \%$

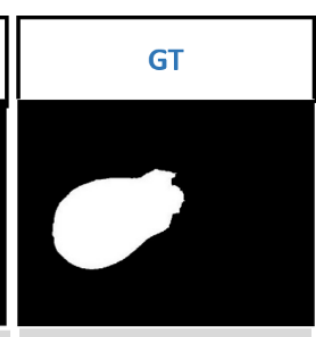

Dice, liver $=100 \%$

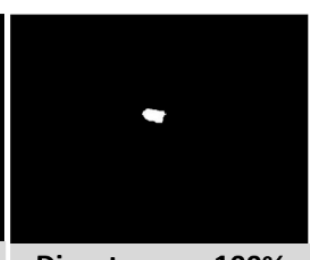

Dice, tumor $=\mathbf{1 0 0 \%}$

Figure 6. A "3D Narrow-band" sample segmentation results. First column contains the input and GT segmentation. Second, third, Forth and last columns provides the results of Model 1, Model 2, proposed FFN, and GT segmentation, respectively; liver (first row) and the tumor (second row)

\subsection{Comparative results}

Results are compared to the related state-of-the-art methods on the LITS competition database to quantify the proposed system strength as shown in Table 2. The proposed FNN fusion system achieves superior performance for tumor segmentation, evidenced by the highest "per case" DSC and the smallest "per case" ASSD among all the compared methods. However, the liver segmentation results are less than the related models. The clinical importance of the accurate liver segmentation is less important than the accurate tumor segmentation, e.g., when considering the case of assisting the radiologists in liver cancer cases. Later, an investigation of how to increase the performance will be introduced, especially for the liver segmentation.

Table 2. Comparative results between the proposed system and the related state-of-the-art methods using the

\begin{tabular}{|c|c|c|c|c|}
\hline \multirow[t]{2}{*}{ Paper } & \multirow[t]{2}{*}{ Experimental setup } & \multirow[t]{2}{*}{ Method } & \multicolumn{2}{|c|}{ "Per case" DSC } \\
\hline & & & Liver & Tumor \\
\hline Bi et al. [10] & $\begin{array}{c}\text { Train size }=118 \\
\text { Test size }=13\end{array}$ & $\begin{array}{l}\text { Cascaded ResNet } \\
\text { (Multi-scale Fusion) }\end{array}$ & $95.1 \%$ & $50.1 \%$ \\
\hline Elmenabawy et al. [28] & $\begin{array}{c}\text { 4-fold validation Train } \\
\text { size }=97 \text { Test size }=33\end{array}$ & $\begin{array}{l}\text { FCN-Alexnet with } \\
\text { preprocessing }\end{array}$ & $90.4 \%$ & $62.4 \%$ \\
\hline Proposed framework & $\begin{array}{c}\text { 5-fold validation } \\
\text { Train size }=26 \\
\text { Test size }=104\end{array}$ & $\begin{array}{l}\text { Fusing Densenet and } \\
\text { FCN-Alexnet }\end{array}$ & $93.5 \%$ & $74.40 \%$ \\
\hline
\end{tabular}




\section{CONCLUSION}

In this paper, a CAD system for simultaneous liver and tumor segmentation is presented, based on the efficient fusion of two deep learning CNN models, trained using 3D narrow-band data. The system performance is evaluated on the challenging LITS database, achieving superior performance over competing methods for liver tumor segmentation. In the future, different CNN architectures as well as different fusion models will be investigated to improve the segmentation accuracy.

\section{REFERENCES}

[1] R. L. Siegel, K. D. Miller, and A. Jemal, "Cancer statistics," CA: A Cancer Journal for Clinicians, vol. 69, pp. 7-34, 2019, doi: $10.3322 /$ caac. 21551.

[2] L. Huang, M. Weng, H. Shuai, Y. Hang, J. Sun, and F. Gao, "Automatic liver segmentation from CT images using single-block linear detection," Biomed Research International, vol. 2016, pp. 1-11, 2016, doi: 10.1155/2016/9420148.

[3] E. Bal, E. Klang, M. Amitai, and H. Greenspan, "Automatic liver volume segmentation and fibrosis classification," Medical Imaging 2018: Computer-Aided Diagnosis, International Society for Optics and Photonics, 2018, vol. 10575, Art. no. 1057506, doi: $10.1117 / 12.2294555$.

[4] M. Barstugan, R. Ceylan, M. Sivri, and H. Erdoğan, "Automatic liver segmentation in abdomen CT images using SLIC and AdaBoost algorithms," 8th International Conference on Bioscience. Biochemistry and Bioinformatics, 2018, pp. 29-133, doi: $10.1145 / 3180382.3180383$.

[5] J. Muthuswamy and B. Kanmani, "Optimization based liver contour extraction of abdominal ct images," International Journal of Electrical and Computer Engineering (IJECE), vol. 8, no. 6, pp. 5061-5070, 2018, doi: 10.11591/ijece.v8i6.pp.5061-5070.

[6] C. C. Chang et al., "Computer-aided diagnosis of liver tumors on computed tomography images," Computer Methods and Programs in Biomedicine, vol. 145, pp. 45-51, 2017, doi: 10.1016/j.cmpb.2017.04.008.

[7] G. Chlebus, A. Schenk, J. H. Moltz, B. V. Ginneken, H. K. Hahn, and H. Meine, "Automatic liver tumor segmentation in CT with fully convolutional neural networks and object-based postprocessing," Scientific reports, vol. 8, no. 1, 2018, Art. no. 15497, doi: 10.1038/s41598-018-33860-7.

[8] O. Ronneberger, P. Fischer, and T. Brox, "U-net: Convolutional networks for biomedical image segmentation," Proceeding of International Conference on Medical Image Computing and Computer, vol. 9351, 2015, pp. 234-241, doi: 10.1007/978-3-31924574-4_28.

[9] Y. Yuan, "Hierarchical convolutional-deconvolutional neural networks for automatic liver and tumor segmentation," arXiv preprint arXiv:1710.04540, 2017.

[10] L. Bi, J. Kim, A. Kumar, and D. Feng, "Automatic liver lesion detection using cascaded deep residual networks," 2017, arXiv preprint arXiv:1704.02703.

[11] N. Gruber et al., "A joint deep learning approach for automated liver and tumor segmentation," 2019, arXiv preprint arXiv:1902.07971.

[12] J. Wang, Y. Cheng, C. Guo, Y. Wang, and S. Tamura, "Shape-intensity prior level set combining probabilistic atlas and probability map constrains for automatic liver segmentation from abdominal CT images," International journal of computer assisted radiology and surgery, vol. 11, no. 5, pp. 817-826, 2019, doi: 10.1007/s11548-015-1332-9.

[13] C. Shi., Y. Cheng, F. Liu, Y. Wang, J. Bai, and S. Tamura, "A hierarchical local region-based sparse shape composition for liver segmentation in CT scans," Pattern Recognition, vol. 50, pp. 88-106, 2016, doi: 10.1016/j.patcog.2015.09.001.

[14] L. I. Song, K. F. Geoffrey, and H. E. Kaijian, "Bottleneck feature supervised U-Net for pixel-wise liver and tumor segmentation," Expert Systems with Applications, vol. 145, pp. 113-131, 2020, doi: 10.1016/j.eswa.2019.113131.

[15] Y. Zhu and S. Newsam, "Densenet for dense flow," 2017 IEEE International Conference on Image Processing (ICIP), 2017, pp. 790-794, doi: 10.1109/ICIP.2017.8296389.

[16] J. Long, E. Shelhamer, and T. Darrell, "Fully convolutional networks for semantic segmentation," Proc. of the IEEE Conference on Computer Vision and Pattern Recognition, 2015, pp. 3431-3440.

[17] A. Mittal, R. Hooda, and S. Sofat, "LF-SegNet: A fully convolutional encoder-decoder network for segmenting lung fields from chest radiographs," Wireless Personal Communications, vol. 10, pp. 511-529, 2018, doi: 10.1007/s11277-018-5702-9.

[18] A. Kalinovsky and V. Kovalev, "Lung image Segmentation using deep learning methods and convolutional neural networks," XIII Int. Conf. in Pattern Recognition and Information Processing, 2016, pp. 21-24.

[19] A. Elnakib, H. M. Amer, and F. E. Abou-Chadi, "Computer aided detection system for early cancerous pulmonary nodules by optimizing deep learning features," Proceedings of the 2019 8th International Conference on Software and Information Engineering, 2019, pp. 75-79, doi: 10.1145/3328833.3328856.

[20] A.A. Moustafa., A. A. Elnakib, and N. F. Areed, "Optimization of deep learning features for age-invariant face recognition," International Journal of Electrical and Computer Engineering (IJECE), vol. 10, no. 2, pp. 1833-1841, 2020, doi: 10.11591/ijece.v10i2.pp1833-1841.

[21] S. E. Nassar, M. A. Mohamed, and A. Elnakib, "MRI brain tumor segmentation using deep learning," Mansoura Engineering Journal, vol. 45, no. 4, pp. 45-54, doi: 10.21608/BFEMU.2021.139470.

[22] D. Elsawah, A. Elnakib, and H. E. D. S. Moustafa, "Deep learning grading system for diabetic retinopathy using fundus images," Mansoura Engineering Journal (MEJ), vol. 45, no. 4, pp. 1-8, doi: 10.21608/bfemu.2020.118646.

[23] K. H. Zou et al., "Statistical validation of image segmentation quality based on a spatial overlap index1: Scientific reports," Academic Radiology, vol. 11, no. 2, pp. 178-189, 2004, doi: 10.1016/S1076-6332(03)00671-8.

[24] S. Raschka, "An overview of general performance metrics of binary classifier systems," arXiv preprint arXiv:1410.5330, 2014

[25] P. Bilic et al., "The liver tumor segmentation benchmark (LITs)," arXiv preprint arXiv:1901.04056, 2019.

[26] P. Christ. "LiTS-liver tumor segmentation challenge." The LITS website. competitions.codalab.org. 2017. https://competitions.codalab.org/competitions/17094 (accessed Aug. 2, 2021).

[27] J. Duchi, E. Hazan, and Y. Singer, "Adaptive subgradient methods for online learning and stochastic optimization," Journal of machine learning research, vol. 12, no. 7, pp. 2121-2159. 2011.

[28] N. A. Elmenabawy, A. Elnakib, and H. Moustafa, "Deep joint segmentation of liver and cancerous nodules from CT images," 2020 37th National Radio Science Conference (NRSC), 2020, pp. 296-301, doi: 10.1109/NRSC49500.2020.9235097. 


\section{BIOGRAPHIES OF AUTHORS}

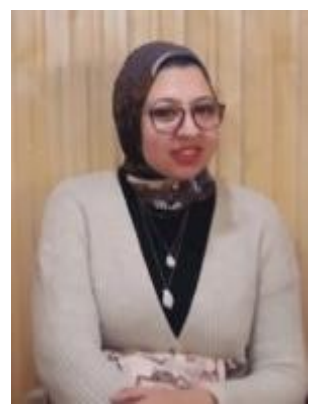

Nermeen Elmenabawy (iD SC SC $P$ is a graduate student in Electronics and Communications Engineering (ECE) Department, Faulty of Engineering, Mansoura University since 2017. She received her BSc from the ECE Department in 2013. She has gained a two-year hand-on experience on medical data analysis during her research studies. She can be contacted at email: eng_nermeena@yahoo.com.

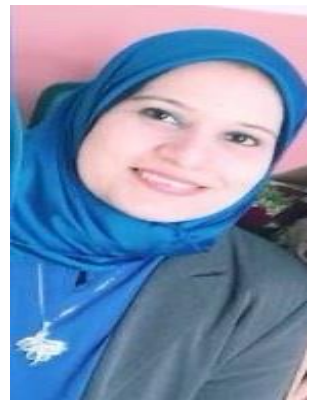

Mervat El-Seddek (iD 8. SC P is an assistant Professor at the Department of Communications and Electronics Engineering, at the higher institute of engineering and technology, Mansoura. The main research points include image processing and machine learning. She can be contacted at email: mervat.elseddek@ieee.org.

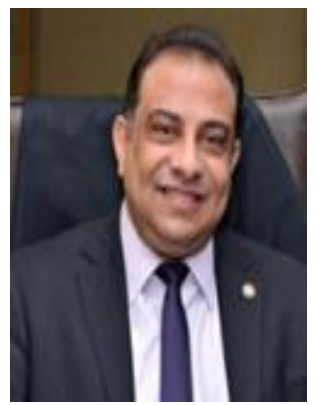

Hossam El-Din Moustafa (D) SC P is an associate Professor at the Department of Electronics and Communications Engineering, and the founder and executive manager of Biomedical Engineering Program (BME) at the Faculty of Engineering, Mansoura University. The main research points include biomedical imaging, signal processing, and deep learning. He can be contacted at email: hossam_moustafa@hotmail.com.

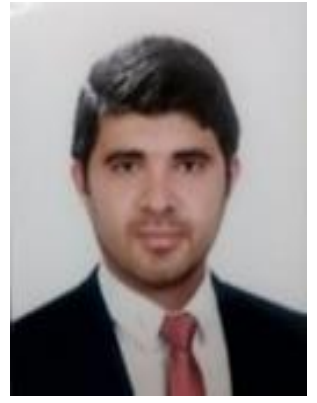

Ahmed Elnakib (D) SC $\mathrm{P}$ is an associate professor in ECE department, Mansoura University. D. Elnakib have authored or co-authored more than 25 journal articles, 7 book chapters, and 30 peer-reviewed conference papers. D. Elnakib is a regular reviewer for top international medical signal analysis journals that include: Medical Image Analysis, IEEE Transactions on Medical Imaging, and Neurocomputing. In 2013, he has awarded the John Houchens Prize for the best outstanding dissertation. He can be contacted at email: nakib@mans.edu.eg. 